\title{
Processamento Cognitivo no Transtorno de Estresse Pós-Traumático: Um Estudo Teórico
}

\author{
Gabriela Sbardelloto, Luiziana Souto Schaefer, Beatriz de Oliveira Meneguelo Lobo, \\ Renato Maiato Caminha \& Christian Haag Kristensen" \\ Pontifícia Universidade Católica do Rio Grande do Sul, Porto Alegre, Brasil
}

\begin{abstract}
RESUMO
O presente artigo discute o processamento cognitivo no Transtorno de Estresse Pós-Traumático (TEPT) através das teorias mais relevantes relacionadas ao desenvolvimento e manutenção do TEPT: Teoria dos Dois Fatores, Teoria do Processamento Emocional, Teoria da Representação Dual e Teoria Cognitiva de Ehlers e Clark. A partir disso, discute-se a relação entre o processamento cognitivo da experiência traumática e o desencadeamento, a manutenção e o agravamento dos sintomas póstraumáticos. As abordagens apresentam modelos explicativos sobre o processamento da memória traumática, enfatizando fatores que ocorrem antes, durante e depois do trauma. Verifica-se a necessidade de pesquisas empíricas acerca do processamento cognitivo no TEPT, esforço que vem a contribuir com hipóteses explicativas mais precisas que implicam em intervenções efetivas no TEPT.
\end{abstract}

Palavras-chave: transtorno de estresse pós-traumático; processamento cognitivo; implicações clínicas.

\begin{abstract}
Cognitive Processing in Posttraumatic Stress Disorder: A Theoretical Study

This article discusses the cognitive processing in Posttraumatic Stress Disorder (PTSD), through the most relevant theories of the development and maintenance of PTSD: Theory of Two Factors, Emotional Processing Theory, Theory of Dual Representation and Ehlers and Clark's Cognitive Theory. Based on this background, the relationship between the cognitive processing of traumatic experience and the onset, maintenance and aggravation of post-traumatic symptoms is discussed. It was found that these theories have explanatory models about the processing of traumatic memories and stress factors that occur before, during and after the traumatic event. There is a need for empirical research on cognitive processing in PTSD, an effort that is contributing to more precise hypotheses imply that effective interventions for PTSD.
\end{abstract}

Keywords: posttraumatic stress disorder; cognitive processing; clinical implications.

A ocorrência de eventos estressores na vida dos indivíduos é bastante comum. Estima-se que em torno de 60 a $90 \%$ da população já sofreu ou será exposta a experiências estressoras traumáticas, sendo que uma parcela considerável dessas pessoas desenvolve o TEPT (Breslau, 2002; Creamer, Burgess, \& McFarlane, 2001; Kessler, Sonnega, Bromet, Hughes, \& Nelson, 1995; Kessler, Chiu, Demler, Jin, \& Walters, 2005). O número crescente de publicações a respeito do transtorno, principalmente nos Estados Unidos, Israel e alguns países da Europa e Oceania evidencia o interesse nessa área (Câmara Filho \& Sougey, 2001).
Infelizmente, no Brasil, o TEPT ainda é pouco estudado, o que acarreta dificuldades significativas em termos de diagnóstico, prevalência e tratamento na população (Bucasio et al., 2005).

De acordo com o Manual Diagnóstico e Estatístico de Transtornos Mentais (DSM-IV-TR), o TEPT pode ser caracterizado, basicamente, como uma síndrome que tem como principais sintomas cognições involuntárias e intrusivas, decorrentes da vivência de um evento estressor traumático (American Psychiatric Association [APA], 2002). Esse fenômeno cognitivo involuntário inclui flashbacks, pesadelos e recorda-

Endereço para correspondência: Christian Haag Kristensen - christian.kristensen@ pucrs.br 
ções intrusivas a respeito da experiência traumática (Taylor, 2006).

Entre as consequências do TEPT, podemos sumarizar um grande impacto psicológico no self, influenciando no desenvolvimento de capacidades de intimidade, amor, vinculação e sexualidade (Briere \& Spinazzola, 2009; Schiraldi, 1999). Quando não tratado, o TEPT está associado a altas taxas de depressão, outros transtornos de ansiedade, suicídio, baixa autoestima, transtornos de personalidade, isolamento social, disfunção sexual, culpa, transtornos dissociativos, relações destrutivas, entre outros (Briere \& Spinazzola, 2009; Kessler et al., 1995; 2005; Schiraldi, 1999).

Atualmente, sabe-se que as vulnerabilidades do indivíduo e o processamento cognitivo do trauma são fatores tão ou mais importantes que o evento estressor em si (Brewin, Andrews, Valentine, 2000; Taylor, 2006). Dessa maneira, diferentes abordagens teóricas têm sido empregadas numa tentativa de explicar as implicações do processamento cognitivo da experiência traumática para o desencadeamento, a manutenção e o agravamento dos sintomas traumáticos. Este artigo teórico teve como principal objetivo discutir o processamento cognitivo no TEPT com o embasamento das principais teorias que abordam o tema, através de uma revisão não sistemática da literatura.

\section{TEORIAS PSICOLÓGICAS DO TEPT}

\section{Teoria dos Dois Fatores}

A Teoria dos Dois Fatores (ou Teoria Bifatorial) sugerida por Mowrer (1960) surgiu de uma adaptação das teorias de condicionamento propostas para a aquisição e a manutenção de outros transtornos de ansiedade (Brewin \& Holmes, 2003). Partindo desse pressuposto, o desenvolvimento do TEPT se dá através de um processo de condicionamento clássico, em que o trauma é um estímulo incondicionado (EI) que gera uma resposta incondicionada (RI) de medo e evitação (Astin \& Resick, 2003). Há, normalmente, um pareamento de outros estímulos neutros que estavam presentes durante o trauma com o evento estressor traumático (Keane, Zimering, \& Caddell, 1985). Dessa maneira, esses estímulos neutros passam a desencadear a resposta de medo (resposta condicionada - RC), tornando-se, então, o estímulo neutro um estímulo condicionado (EC).

A manutenção do transtorno, por sua vez, ocorre por meio de condicionamento operante, processo no qual os estímulos neutros transformam-se em estímu- los condicionados, produtores de resposta de medo intenso e evitação (RC). Assim, seria esperado que, com a cessação da apresentação do evento estressor (EI), a associação entre EC e RC fosse extinta. No entanto, o intenso medo faz com que se evite a lembrança ou as situações associadas ao evento estressor (EI) e à resposta de ansiedade (RC), gerando alívio momentâneo. Desse modo, a evitação da lembrança do trauma ou de situaçõos associadas (EC) passa a ser reforçada negativamente, impedindo a extinção da associação entre a lembrança do trauma (com seus estímulos desencadeadores) e resposta de ansiedade (RC) (Mowrer, 1960).

Dessa maneira, esta teoria pressupõe que estímulos neutros passam a se transformar em estímulos condicionados que geram respostas condicionadas cada vez mais fortalecidas pela evitação (Mowrer, 1960). No condicionamento operante, através da generalização do estímulo, é possível explicar como os sintomas frequentemente pioram durante o tempo, acarretando num aumento da evocação de memórias traumáticas e excitabilidade fisiológica (Keane et al., 1985). Assim, a teoria permite entender como o sistema de respostas de esquiva é mantido, gerado pelo aumento da ansiedade (Knapp \& Caminha, 2003). Por outro lado, pesquisadores abordam que a Teoria dos Dois Fatores é incompleta e parcimoniosa para esclarecer os sintomas do TEPT, não fornecendo uma explicação para a grande generalização de estímulos evitados pelos pacientes com esse diagnóstico (Cahill \& Foa, 2007; Taylor, 2006). Além disso, Cahill e Foa (2007) argumentam que o problema maior para essa abordagem reside em explicar convincentemente os sintomas de reexperiência, particularmente pensamentos intrusivos, flashbacks e pesadelos.

\section{Teoria do Processamento Emocional}

Acredita-se que emoções extremas experienciadas durante um trauma conduzem a um processamento da informação de eventos traumáticos que produz desorganização e recordações de memória incoerentes com a memória traumática (Foa \& Riggs, 1993). A Teoria do Processamento Emocional parte da premissa de que os transtornos de ansiedade refletem a presença de estruturas de medo patológico na memória, ou seja, representações de estímulos inofensivos que são erroneamente associados com o significado de perigo, conduzindo a uma percepção generalizada de ameaça (Cahill \& Foa, 2007; Foa \& Rothbaum, 1998). Além disso, as memórias traumáticas codificam uma larga escala de respostas fisiológicas e comportamentais que podem estar ligadas a uma avaliação da falta de 
efetividade pessoal face à ameaça. Essa avaliação leva a uma representação generalizada do self como totalmente inapto (Brewin \& Holmes, 2003).

Eventos traumáticos modificam as crenças básicas das pessoas, uma vez que há um aumento geral de crenças negativas sobre o self, sobre o mundo e sobre os outros após a vivência do evento traumático (Foa, Steketee \& Rothbaum, 1989). Quando vítimas com TEPT são comparadas a vítimas que não desenvolveram o transtorno, as alterações nessas crenças ficam evidenciadas (Foa, Ehlers, Clark, Tolin \& Orsillo, 1999).

Um dos pontos centrais da teoria é a estrutura de medo, que se mantém por longo tempo na memória. A rede, formada através do condicionamento e da generalização, abrange informações de estímulos eliciados após o evento traumático, informações sobre cognições, comportamentos e reações fisiológicas do trauma e informações que ligam esses estímulos a elementos de resposta (Foa \& Rothbaum, 1998; Foa et al., 1989). A ativação dessa rede de medo se dá, posteriormente, através de lembranças do trauma que trazem informações à rede. Portanto, tentativas de impedir e suprimir tais ativações conduzem a um conjunto de sintomas de evitação (Foa et al., 1989).

Uma das limitações da Teoria do Processamento Emocional é sobre o seu foco no medo como primeira emoção e no perigo como o primeiro significado associado ao TEPT. Ainda que as terapias de exposição baseadas nessa teoria - obtenham bons resultados empíricos, eles não garantem a validade da teoria (Cahill \& Foa, 2007). Outra crítica se refere à possibilidade de modificação de memórias através da correção de informações. Apesar desses pontos a serem considerados, a Teoria do Processamento Emocional é apontada como uma das teorias mais largamente utilizadas e bem articuladas para o TEPT (Taylor, 2006).

\section{Modelo de Representação Dual}

O Modelo de Representação Dual (Brewin et al., 1996) aponta que, diante de um evento traumático, é possível que os indivíduos apresentem dois sistemas de memória que trabalham em paralelo - o sistema de memória verbalmente acessível (VAM) e o sistema de memória situacionalmente acessível (SAM) (Brewin, 2001).

O sistema VAM organiza a informação em estruturas coerentes, em forma de narrativas, estando submetido a um controle mais consciente. Narrativas de memória oral ou escrita do trauma representam uma operação de um sistema de acessibilidade de memória verbal, refletindo o fato de que a memória traumática foi integrada com outras memórias autobiográficas (Brewin et al., 1996).

O sistema SAM é uma memória mais fragmentada, acessado de forma predominantemente automática, englobando informações que não são gravadas no sistema VAM e requer um nível mais baixo de processamento perceptual da informação, o qual não é necessariamente integrado ao conhecimento autobiográfico (Brewin \& Holmes, 2003; Brewin et al., 1996; Taylor, 2006). O conteúdo armazenado no sistema SAM é acessado por estímulos correspondentes no ambiente, sendo difícil paras as pessoas controlarem os gatilhos dessas memórias, já que não estão aptas para evitar essas lembranças. Assim, os sonhos e os flashbacks característicos do TEPT são apontados como produtos do sistema SAM (Brewin \& Holmes, 2003; Taylor, 2006).

A teoria da Representação Dual postula que, à medida que o tempo passa em relação à ocorrência do trauma, as memórias traumáticas tendem a tornar-se mais genéricas e abstratas, e com isso, perdem os detalhes sensoriais que possuem inicialmente (Brewin et al., 1996). Portanto, terapias que envolvam a exposição às recordações relativas ao trauma e que produzem no raciocínio associativo novos padrões e regularidades que abrangem os mesmos elementos que fizeram parte da experiência traumática, facilitam um processo de atualização e reconstrução das memórias traumáticas (Brewin \& Holmes, 2003).

Algumas críticas são apontadas ao modelo de Brewin (1996). Apesar de pessoas com TEPT reportarem períodos em que suas memórias traumáticas encontram-se em forma verbal e períodos que estão em formato de imagens, não há evidências conclusivas de que existam dois sistemas distintos de memória. Diferenças nas condições de codificação e em estratégias de recuperação influenciam a natureza do material armazenado na memória e podem explicar essa distinção de recordações sem a necessidade de sistemas distintos de memória. Ainda, o modelo da representação dual não apresenta evidências convincentes de que o sistema SAM contém informações que não são armazenadas no sistema VAM, e tem poucas explicações sobre como funciona a interação entre esses dois sistemas.

\section{Teoria Cognitiva de Ehlers e Clark}

O modelo cognitivo de Ehlers e Steil (1995) propõe que a inibição da mudança no significado atribuído 
às cognições traumáticas impede a melhora dos sintomas do TEPT. Os autores descobriram que significados idiossincráticos de pensamentos intrusivos experienciados pelas pessoas com TEPT prediziam um alto nível individual de estresse que resultaria em estratégias para controlar esses pensamentos. Ou seja, se um indivíduo percebe os pensamentos intrusivos como um indicador de que está perdendo o controle ou enlouquecendo, a motivação para suprimir esses pensamentos é potencializada (Ehlers \& Steil, 1995). Essa supressão mantém um círculo vicioso, e as estratégias de controle resultam na manutenção desses pensamentos intrusivos, impedindo, assim, mudanças no significado do trauma. Em função disso, Ehlers e Steil (1995) passaram a sugerir uma avaliação detalhada do processo que tende a impedir mudanças no significado do trauma, incluindo comportamentos de segurança, supressão de memórias e pensamentos que relatem o trauma e o processamento de seleção de informações (Gray, Maguen, \& Litz, 2007).

A Teoria Cognitiva de Ehlers e Clark (2000) é um dos modelos mais complexos para explicar o funcionamento cognitivo no TEPT, englobando conceitos e pressupostos de outros modelos, como condicionamento, crenças disfuncionais, esquemas e memórias verbais e situacionais. O modelo dá ênfase aos fatores que são responsáveis pela manutenção do TEPT (Taylor, 2006).

Duas proposições-chave conduzem a um sentido de ameaça atual: (a) diferenças individuais na avaliação do trauma e/ou de suas sequelas; (b) diferenças individuais na natureza da memória para o evento e sua ligação a outras memórias autobiográficas (Ehlers \& Clark, 2000). Uma vez ativada, a percepção da ameaça atual é acompanhada de intrusões e de outros sintomas de revivência, sintomas de hipervigilância, ansiedade e outras respostas emocionais. Esse senso de ameaça também motiva uma série de respostas de comportamentos e cognições, que pretendem reduzir a ameaça percebida e o estresse em curto prazo, tendo como consequência o impedimento de mudanças cognitivas e a manutenção do transtorno (Brewin \& Holmes, 2003; Dalgleish, 2004; Ehlers \& Clark, 2000; Ehring, Ehlers, \& Glucksman, 2006).

Assim, o TEPT se cronifica no indivíduo traumatizado quando ele processa cognitivamente o evento traumático como uma ameaça atual, o que produz medo e evitação relacionados aos estímulos que lembram o trauma (Ehlers \& Clark, 2000). Tais pensamentos podem ser relacionados ao desenvolvimento de TEPT, sendo que as pessoas que irão desenvolver o transtorno serão aquelas que, durante o evento traumático, processam o significado e as implicações do evento sofrido como um senso de ameaça atual como se não houvesse separação temporal entre presente, passado e futuro. Esse tipo de reação peritraumática está associado com um distúrbio na memória autobiográfica, caracterizado, basicamente, por baixa elaboração e conceitualização, associações erradas de memória e erros no priming perceptual. Esses aspectos impedem a pessoa de situar o trauma no passado e implicam em avaliações negativas, nas quais a possibilidade de que eventos aversivos voltem a ocorrer é superestimada e, portanto, a competência do self passa a ser subestimada (Brewin \& Holmes, 2003; Dalgleish, 2004; Ehlers \& Clark, 2000). Outros fatores peritraumáticos implicam em: incapacidade de estabelecer uma perspectiva autorreferencial durante a vivência do trauma, dissociação, entorpecimento emocional e falta de capacidade cognitiva para avaliar aspectos sobre o evento de maneira acurada (Brewin \& Holmes, 2003).

As avaliações negativas incluem interpretações particulares sobre o trauma relacionadas às consequências, ao mundo, aos outros e à avaliação dos sintomas de TEPT. Essas avaliações errôneas explicam a variedade de emoções subsequentes, como ansiedade, raiva, vergonha e culpa, as quais, por sua vez, motivam as pessoas a se engajarem em estratégias de defesa desadaptativas. Num primeiro momento, essas estratégias ajudam a reduzir o estresse a curto prazo, mas, a longo prazo, acabam por perpetuar o ciclo de ameaça (Brewin \& Holmes, 2003; Ehlers \& Clark, 2000). Além disso, as experiências e crenças pessoais prévias influenciam os tipos de avaliações errôneas realizadas pelos indivíduos com TEPT (Brewin \& Holmes, 2003).

O modelo também explica o desenvolvimento de fortes associações condicionadas entre estímulos (E) e respostas (R) e entre os elementos do evento (estímulo-estímulo) (Dalgleish, 2004), propondo que o problema mais persistente no TEPT é que as associações entre os E-R estão particularmente erradas. Isso faz com que a provocação de memórias do evento e/ou respostas emocionais sejam associadas mais facilmente a estímulos (Ehlers \& Clark, 2000). A memória do trauma e as avaliações de ameaça são ditas como mutuamente influenciadas por outras e estão diretamente relacionadas com a maneira pela qual a informação é processada durante o trauma (Halligan, Michael, Clark, \& Ehlers, 2003). A recuperação de uma memória pode conduzir ou reforçar avaliações de perigo ou 
de ameaça relevante, de forma que torna-se mais fácil recordar memórias congruentes com a situação vivenciada. A baixa elaboração de memórias é também responsável pela dificuldade em integrar as informações que podem impedir que avaliações negativas sejam conduzidas. Ruminações deliberadas utilizadas na tentativa de entender o trauma podem igualmente indicar a perpetuação do TEPT (Ehlers \& Clark, 2000; Dalgleish, 2004).

Diversos estudos testaram a predição dos fatores cognitivos postulados pela Teoria Cognitiva de Ehlers e Clark (2000) em relação ao desenvolvimento e manutenção do TEPT (Dunmore, Clark, \& Ehlers, 2001; Halligan, et al., 2003; Kleim, Ehlers, \& Glucksman, 2007). Ao avaliar profissionais que trabalhavam em emergência hospitalar, um estudo observou que a forma como os sujeitos da amostra avaliaram o evento traumático, a sua sequela e a dissociação peritraumática predisseram sintomas mais severos do transtorno. Ao controlar a frequência dos sintomas intrusivos, o estudo pôde confirmar a teoria de que o uso da ruminação e supressão pode, inadvertidamente, manter o TEPT, impedindo mudanças nas avaliações negativas e na memória traumática (Laposa \& Alden, 2003).

As variáveis cognitivas envolvendo avaliação negativa do trauma e estratégias cognitivas disfuncionais empregadas após o evento apresentaram correlações significativas com a intensidade da sintomatologia do TEPT, predizendo, inclusive, a gravidade do transtorno de 3 a 6 meses após o evento traumático (Ehlers, Mayou, \& Bryant, 2001). O presente modelo cognitivo do TEPT apresentou ainda predições estatísticas e elevado grau de variância dos sintomas fóbicos e depressivos em vítimas de acidentes automotores (Ehring et al., 2006).

De maneira geral, pode-se afirmar que, além da viabilidade empírica dos postulados apresentados pela Teoria Cognitiva de Ehlers e Clark (2000), esse modelo apresenta construtos incluídos em outros modelos teóricos. Esse aspecto confirma o poder exploratório da teoria, que é considerada tão boa ou até melhor que outros modelos explicativos (Taylor, 2006).

\section{PROCESSAMENTO COGNITIVO E O DESENVOLVIMENTO DO TEPT}

Os modelos teóricos anteriormente descritos enfatizam, em grande parte, o processamento cognitivo pós-traumático, visto que os próprios modelos foram propostos para explicar a aquisição e a manutenção do TEPT. Em comum, essas abordagens apresentam algum modelo explicativo sobre o processamento da memória traumática e sobre as alterações em termos dos conteúdos da cognição (por exemplo, crenças) após a ocorrência do evento traumático. Ainda estão presentes, em menor grau, propostas sobre o funcionamento (vulnerabilidade psicológica) e conteúdos que antecedem (por exemplo, cognições pré-traumáticas) ou ocorrem simultaneamente (cognições peritraumáticas) ao evento estressor (Keane, Marshall, \& Taft, 2006; Bryant \& Guthrie, 2007; Ozer \& Weiss, 2004).

Tendo em vista os aspectos discutidos acerca dos modelos explicativos do processamento cognitivo do TEPT, esta seção busca discutir a relação entre o processamento cognitivo da experiência traumática e o desencadeamento, a manutenção e o agravamento dos sintomas pós-traumáticos. Há evidências de que o TEPT seja parcialmente mediado pelas respostas emocionais, comportamentais e fisiológicas decorrentes do sistema de crenças associados à experiência traumática (Ehlers \& Clark, 2000; Foa \& Riggs, 1993; Foa \& Rothbaum, 1998). Dessa forma, destacam-se o papel dos processos e conteúdos cognitivos que ocorrem antes, durante e após um evento traumático, que estão fortemente associados ao desenvolvimento e à manutenção do TEPT (Keane et al., 2006; Ozer \& Weiss, 2004; Yehuda, 1999).

No processamento cognitivo pré-traumático, estão incluídas as crenças prévias ao evento estressor traumático. Crenças muito rígidas anteriores ao trauma tanto negativas quanto positivas - sobre o self e o mundo tornam os indivíduos mais vulneráveis a desenvolver TEPT (Foa \& Rothbaum, 1998). Modelos cognitivos como o de Foa et al. (1999) sustentam que o TEPT é mantido basicamente por dois tipos de crenças negativas: pensamentos extremos sobre a periculosidade do mundo e sobre o self como incompetente (Foa \& Riggs, 1993; Foa \& Rothbaum, 1998).

Quando o desenvolvimento do TEPT está relacionado às crenças rígidas negativas, o conteúdo das cognições caracteriza-se por interpretações catastróficas sobre ameaças e por crenças de inabilidade em lidar com essas ameaças. Esses indivíduos que veem a si mesmos como incompetentes e o mundo como perigoso têm tais premissas confirmadas e reforçadas quando experienciam o evento traumático (Taylor, 2006). Ao mesmo tempo, os indivíduos que possuem crenças rígidas e positivas sobre o self como altamente competente e sobre o mundo como altamente seguro também têm essas crenças impactadas e desorganizadas após experienciarem o evento estressor, já que a dimensão do evento é altamente contraditória às cren- 
ças prévias (Brewin \& Holmes, 2003). Assim, independentemente das crenças serem positivas ou negativas, quando são estanques, fazem com que o indivíduo passe a ver-se como totalmente incompetente e o mundo como altamente perigoso (Foa et al., 1989). Por outro lado, pessoas que têm crenças mais flexíveis sobre graus de competência e segurança são vistas como mais resilientes aos efeitos do trauma, pois elas são mais hábeis a interpretar o trauma como uma experiência incomum, sem maiores implicações às crenças sobre o self e o mundo existentes até então (Brewin \& Holmes, 2003; Foa et al., 1989; Taylor, 2006).

Uma pesquisa conduzida com bombeiros antes de terem sido expostos a eventos estressores foi a primeira confirmação empírica de que autoavaliações rígidas e catastróficas prévias a experiências traumáticas predizem uma maior vulnerabilidade ao TEPT, corroborando modelos cognitivos anteriores (Bryant \& Guthrie, 2007; Foa et al., 1999). Em estudo com vítimas de agressão, constatou-se, após follow-up de 6 e 9 meses, que variáveis cognitivas como crenças negativas sobre o self e sobre o mundo predisseram significativamente a gravidade de sintomas de TEPT (Dunmore et al., 2001).

As cognições peritraumáticas dizem respeito ao processamento cognitivo que ocorre durante o evento traumático e estão diretamente relacionadas à percepção de ameaça à vida do indivíduo exposto ao trauma. A dissociação peritraumática inclui sintomas referentes ao processamento cognitivo no momento da vivência traumática e evolve alteração no senso de realidade (irrealização), alteração no sentido de passagem do tempo e sentimentos de desconexão com o próprio corpo (despersonalização) (Birmes et al., 2003; Keane et al. 2006). Um estudo de metanálise encontrou alta correlação entre dissociação peritraumática e o desenvolvimento e a manutenção do TEPT (Ozer, Best, Lipsey, \& Weiss, 2003). A força dessa correlação parece não diferir em função do tipo de trauma experienciado, mas varia em função do tempo decorrido entre o trauma e a avaliação dos sintomas, do tipo de amostra e do método utilizado para a avaliação dos sintomas (Ozer et al., 2003; Ozer \& Weiss, 2004).

O processamento cognitivo que ocorre após a exposição ao trauma, ou seja, as cognições póstraumáticas são amplamente descritas na literatura como preditoras do desenvolvimento e manutenção do TEPT (Bryant \& Guthrie, 2005; Stallard \& Smith, 2007). Esses achados são condizentes à ideia de que o trauma traz sobre o self uma mudança negativa e permanente. (Dunmore, Clark \& Ehlers, 1999; Ehlers, Mayou \& Bryant, 1998; 2001).
No entanto, não é possível concluir que as variáveis pré-traumáticas não são importantes ou, ainda, menos importantes que as variáveis peritraumáticas e pós-traumáticas na manutenção do TEPT. O processamento cognitivo e as avaliações de um evento traumático são influenciados por características do evento e por fatores que ocorrem previamente à ocorrência de um evento traumático, como consumo de álcool, baixa inteligência e crenças e experiências prévias (Brady, Back, \& Coffey, 2004; Ehlers \& Clark, 2000; Green et al., 2010; Kremen et al., 2007; O'Donnell et al., 2009).

\section{IMPLICAÇÕES CLÍNICAS}

A avaliação do transtorno tem dois objetivos fundamentais: diagnóstico e auxilio terapêutico. Atualmente, sabe-se que o TEPT apresenta alta comorbidade com outros transtornos (Kessler et al., 1995; 2005), por isso, a correta constatação do diagnóstico faz com que se tenha um olhar mais atentos para outros futuros problemas psicológicos. Ainda no que tange à avaliação, ressalta-se a importância de identificar as cognições pós-traumáticas disfuncionais, uma vez que elas podem influenciar diretamente na gravidade e na manutenção dos sintomas de TEPT (Foa et al., 1999; Stallard \& Smith, 2007).

A identificação das crenças distorcidas póstraumáticas permite, através da Terapia CognitivoComportamental, que seja realizada uma reestruturação cognitiva, ou seja, a modificação das interpretações distorcidas dos fatos (Beck, 2005). No TEPT, objetiva-se a modificação dos esquemas e crenças que estão imbricados na manutenção do transtorno, através de técnicas como o registro de pensamentos disfuncionais (RPD) e questionamento socrático, para que os pacientes identifiquem seus padrões de pensamentos disfuncionais e possam modificar o significado do evento traumático pelo qual passaram e suas crenças desadaptativas (Rothbaum, Meadows, Resick, \& Foy, 2000; Beck, 1997; Kristensen, Schaefer, \& Mello, 2011).

A reestruturação cognitiva no TEPT consiste em modificar, principalmente, as crenças distorcidas sobre o self e mundo, já que, conforme revisado pela Teoria do Processamento Emocional (Foa et al., 1989), essas cognições estão diretamente relacionadas ao desenvolvimento e à manutenção do transtorno. Ainda, a reestruturação cognitiva atua no processamento cognitivo apontado pela Teoria Cognitiva de Ehlers e Clark (2000), na qual o TEPT está relacionado a avaliações negativas quanto à ocorrência de novos eventos traumáticos, quanto à baixa competência 
do self em lidar com esses eventos, quanto a interpretações específicas sobre o trauma e suas consequências e quanto à avaliação dos sintomas de TEPT.

As intervenções baseadas em exposição aos estímulos do trauma vêm recebendo amplas evidências empíricas (Moser, Cahill, \& Foa, 2010) e têm por objetivo reduzir a ansiedade vinculada aos estímulos do trauma associada aos sintomas de revivência e evitação e organizar as memórias traumáticas (Keane et al., 2006). Segundo a Teoria Cognitiva de Ehlers e Clark (2000), o TEPT é um transtorno marcado por memórias traumáticas condicionadas erroneamente, que por sua vez, apresentam papel fundamental no processamento das informações. Ainda, a Teoria do Processamento Emocional de Foa e cols. (1989) sugere que um tratamento efetivo para o TEPT requer a exposição aos estímulos traumáticos, seja através de exposição imagística, na qual ocorre um enfraquecimento da ligação entre os estímulos relacionados ao trauma e a ativação emocional condicionada; ou através da exposição situacional, que ensina aos pacientes que certos estímulos não são perigosos, ao incorporar informações de segurança na estrutura de medo. Assim, através da confrontação de situações, pensamentos, atividades e lembranças que ativem medo e comportamentos de evitação, o paciente é estimulado a enfrentar o medo e confrontar a sintomatologia associada à revivência e evitação, além de possibilitar a reorganização das memórias traumáticas (Foa \& Rothbaum, 1998; Kristensen et al., 2011; Riggs, Cahill, \& Foa, 2006).

\section{CONSIDERAÇÕES FINAIS}

Entender o funcionamento cognitivo no TEPT é válido para auxiliar no tratamento de pessoas que sofreram algum trauma. No entanto, são necessárias pesquisas a fim de clarificar como se dá a manutenção dos esquemas disfuncionais em diferentes populações, para que, dessa forma, seja possível auxiliar com intervenções adequadas. Sabe-se que intervenções apropriadas com relação às crenças disfuncionais reduzem a ansiedade, diminuem a valência dos esquemas desadaptativos e aumentam a capacidade de metacognição (McNally, 2003).

Os modelos teóricos aqui apresentados divergem em importantes aspectos, assim como apresentam diferentes limitações e direções futuras. Destaca-se o importante papel da Teoria Cognitiva de Ehlers e Clark e da Teoria do Processamento Emocional na fundamentação teórica de intervenções cognitivo- comportamentais amplamente utilizadas e empiricamente testadas para o TEPT, como a reestruturação cognitiva e terapia de exposição.

Outros modelos, como a Teoria dos Dois Fatores permite compreender a manutenção de comportamentos evitativos, perpetuados pelo aumento da ansiedade, no entanto, é um modelo explicativo falho quanto à compreensão da sintomatologia de revivência. $\mathrm{O}$ Modelo de Representação Dual enfatiza o TEPT como um transtorno da memória, em que os sistemas de memória verbalmente acessível (VAM) e situacionalmente acessível (SAM) sobressaem-se um em relação ao outro. Esse modelo traz importantes avanços ao compreender a fragmentação de memórias dos indivíduos com TEPT, uma vez que essa compreensão possibilita a ênfase na organização das memórias traumáticas; no entanto, não há evidências conclusivas acerca da existência de sistemas distintos de memória. Uma limitação apontada em relação a todas as teorias aqui abordadas é que nenhuma delas aborda como fatores desenvolvimentais podem influenciar o risco e a manifestação do TEPT em crianças (Taylor, 2006). Cabe destacar que nenhuma das abordagens teóricas apresenta uma compreensão definitiva para o TEPT.

De maneira geral, os modelos teórico-explicativos para o desenvolvimento e manutenção dos sintomas de TEPT apresentam ênfase a fatores que ocorrem antes, durante e após a vivência de um evento traumático. Os modelos diferem quanto a elucidações ao início tardio do TEPT; quanto à explicação dos sintomas de entorpecimento; quanto às altas taxas de comorbidades e à associação com raiva, culpa e vergonha, entre outros fatores. As suposições teóricas envolvem o processamento de memórias, crenças e pensamentos pós-traumáticos, além de fatores prétraumáticos, como vulnerabilidades individuais e, por fim, experiências peri-traumáticas. Destaca-se a importância da compreensão do modelo e do funcionamento do TEPT, uma vez que a partir de explanações teóricas acerca da manutenção da sintomatologia póstraumática torna-se possível investigar e empregar estratégias terapêuticas eficazes.

\section{REFERÊNCIAS}

American Psychiatric Association (2002). Manual diagnóstico e estatístico de transtornos mentais (4 ${ }^{\mathrm{a}}$ ed.; Texto Revisado). Porto Alegre: Artmed.

Astin, M. C., \& Resick, P. A. (2003). Tratamento cognitivocomportamental do transtorno de estresse-pós-traumático. Em V. E. Caballo (Ed..), Manual para o tratamento cognitivocomportamental dos transtornos psicológicos: Transtornos de 
ansiedade, sexuais, afetivos e psicóticos (M. L. Pedro, Trans.) (pp. 226-254). São Paulo: Editora Santos.

Beck, A. T. (2005). The current state of cognitive therapy: A 40years retrospective. Archives of General Psychiatry, 62, 953959.

Beck, J. G. (1997). Terapia cognitiva: Teoria e prática (S. Costa, Trad.). Porto Alegre: Artmed.

Birmes, P., Brunet, A., Carreras, D., Ducassé, J.-L., Charlet, J.-P., $\&$ Lauque, D. (2003). The predictive power of peritraumatic dissociation and acute stress symptoms for posttraumatic stress symptoms: A three-month prospective study. American Journal of Psychiatry, 160, 1337-1339.

Brady, K. T., Back, S. E., \& Coffey, S. F. (2004). Substance sbuse and posttraumatic stress disorder. Current Directions in Psychological Science, 13, 206-209.

Breslau, N. (2002). Epidemiologic studies of trauma, posttraumatic stress disorder, and other psychiatric disorders. Canadian Journal of Psychiatry, 47, 923-929.

Brewin, C. R. (2001). A cognitive neuroscience account of posttraumatic stress disorder. Behaviour Research and Therapy, 39, 373-393.

Brewin, C. R., Andrews, B., \& Valentine, J. D. (2000). Metaanalysis of risk factors for posttraumatic stress disorder in trauma-exposed adults. Journal of Consulting and Clinical Psychology, 68, 748-766.

Brewin, C. R., Dalgleish, T., \& Joseph, S. (1996). A dual representation theory of posttraumatic stress disorder. Psychological Review, 103, 670-686.

Brewin, C. R., \& Holmes, E. A. (2003). Psychological theories of posttraumatic stress disorder. Clinical Psychology Review, 23, 339-376.

Briere, J., \& Spinazzola, J. (2009). Assessment of the sequelae of complex trauma. Em C. Courtois \& J. Ford (Eds.), Treating complex traumatic stress disorders: An evidence-based guide (pp. 104-123). New York: Guildford Press.

Bryant, R. A., \& Guthrie, R. M. (2007). Maladaptive selfappraisals before trauma exposure predict posttraumatic stress disorder. Journal of Consulting and Clinical Psychology, 75, 812-815.

Bryant, R. A., \& Guthrie, R. M. (2005). Maladaptive appraisals as a risk factor for posttraumatic stress: A study of trainee firefighters. Psychological Science, 16, 749-752.

Bucasio, E., Vieira, I., Berger, W., Martins, D., Souza, C., Maia, D. et al. (2005). Transtorno de estresse pós-traumático como acidente de trabalho em um bancário: Relato de um caso. Revista de Psiquiatria do Rio Grande do Sul, 27, 86-89.

Cahill, S. P., \& Foa, E. B. (2007). Psychological theories of PTSD. Em M. D. Friedman, T. M. Keane \& P. A. Resick (Eds.), Handbook of PTSD: Science and practice (pp. 55-77). New York: Guilford Press.

Câmara Filho, J. W. S. C., \& Sougey, E. B. (2001). Transtorno de estresse pós-traumático: Formulação diagnóstica e questões sobre comorbidade. Revista Brasileira de Psiquiatria, 23, 221228.

Creamer, M., Burgess P., \& McFarlane, A. C. (2001). Posttraumatic stress disorder: Findings from the Australian National Survey of Mental Health and Well-Being. Psychological Medicine, 31, 1237-1247.
Dalgleish, T. (2004). Cognitive approaches to posttraumatic stress disorder: The Evolution of multirepresentational theorizing. Psychological Bulletin, 130, 228-260.

Dunmore E., Clark D. M., \& Ehlers, A. (2001). A prospective investigation of the role of cognitive factors in persistent posttraumatic stress disorder PTSD after physical or sexual assault. Behavior Research and Therapy, 39, 1063-1084.

Dunmore, E., Clark, D. M., \& Ehlers, A. (1999). Cognitive factors involved in the onset and maintenance of posttraumatic stress disorder (PTSD) after physical or sexual assault. Behaviour Research and Therapy, 37, 809-829.

Ehlers, A., \& Clark, D. M. (2000). A cognitive model of posttraumatic stress disorder. Behaviour Research and Therapy, 38, 319-345.

Ehlers, A., Mayou, R. A., \& Bryant, B. (1998). Psychological predictors of chronic posttraumatic stress disorder after motor vehicle accidents. Journal of Abnormal Psychology, 107, 508519.

Ehlers, A., Mayou, R. A., \& Bryant, B. (2001). Cognitive predictors of posttraumatic stress disorder in children: Results of a prospective longitudinal study. Behavior Research and Therapy, 41, 1-10.

Ehlers, A., \& Steil, R. (1995). Maintenance of intrusive memories in posttraumatic stress disorder: A cognitive approach. Behavioural and Cognitive Psychotherapy, 23, 217-249.

Ehring, T., Ehlers, A., \& Glucksman E. (2006). Contribution of cognitive factors to the prediction of post-traumatic stress disorder, phobia and depression after motor vehicle accidents. Behaviour Research and Therapy 44, 1699-1716.

Foa, E. B., Ehlers, A., Clark, D. M., Tolin, D. F., \& Orsillo, S. M. (1999). The Posttraumatic Cognitions Inventory (PTCI): Development and validation. Psychological Assessment, 11, 303314.

Foa, E. B., \& Riggs, D. S. (1993). Post-traumatic stress disorder in rape vicitims. Em J. Oldham, M. B. Riba \& A. Tasman (Eds.), American Psyachiatric Press review of psychiatry (pp. 285309). Washington, DC: American Psychiatric Press.

Foa, E. B., \& Rothbaum, B. O. (1998). Treating the trauma of rape: Cognitive behavioral therapy for PTSD. New York: Guilford Press.

Foa, E. B., Steketee, G., \& Rothbaum, B. O. (1989). Behavioral/cognitive conceptualization of post-traumatic stress disorder. Behavior Therapy, 20, 155-176.

Gray, M., Maguen, S., \& Litz, B. (2007). Schema constructs and cognitive models of posttraumatic stress disorder. Em L. P. Riso, P. L. du Toit, D. J. Stein e J. E. Young (Eds.), Cognitive schemas and core beliefs in psychological problems: A scientist-practitioner guide. Washington, DC: American Psychological Association.

Green, J. G., McLaughlin, K. A., Berglund, P. A., Gruber, M. J., Sampsom, N. A., Zaslavsky, A. M. et al. (2010). Childhood adversities and adult psychopathology in the National Comorbidity Survey Replication (NCS-R) I: Associations with first onset of DSM-IV disorders. Archives of General Psychiatry, 67, 113-123.

Halligan, S. L., Michael, T., Clark, D. M., \& Ehlers, A. (2003). Posttraumatic stress disorder following assault: The role of cognitive processing, trauma memory, and appraisals. Journal of Consulting and Clinical Psychology 71, 419-431. 
Keane, T. M., Zimering, R. T., \& Caddell, J. M. (1985). A behavioral formulation of posttraumatic stress disorder in Vietnam veterans. Behavior Therapist, 8, 9-12.

Keane, T. M., Marshall, A. D., \& Taft, C. T. (2006). Posttraumatic stress disorder: Etiology, epidemiology, and treatment outcome. Annual Review of Clinical Psychology, 2, 161-197.

Kessler, R. C., Chiu, W. T., Demler, O., Jin, R., \& Walters, E. E. (2005). Prevalence, severity, and comorbidity of 12-Month DSM-IV disorders in the National Comorbidity Survey Replication. Archives of General Psychiatry, 62, 617-627.

Kessler, R. C., Sonnega, A., Bromet, E., Hughes, M., \& Nelson, C. B. (1995). Posttraumatic stress disorder in the National Comorbidity Survey. Archives of General Psychiatry, 52, 1048-1060.

Kleim, B., Ehlers, A., \& Glucksman, E. (2007). Early predictors of chronic post-traumatic stress disorder in assault survivors. Psychological Medicine, 37, 1457-1467.

Knapp, P., \& Caminha, R. M. (2003). Terapia cognitiva do transtorno de estresse pós-traumático. Revista Brasileira de Psiquiatria, 25, 31-36.

Kremen, W. S., Koenen, K. C., Boake, C., Purcell, S., Eisen, S. A., Franz, C. E. et al. (2007). Pretrauma cognitive ability and risk for posttraumatic stress disorder: A twin study. Archives of General Psychiatry, 64, 361-368.

Kristensen, C. H., Schaefer, L. S., \& Mello, P. G. (2011). Modelo cognitivo-comportamental do transtorno de estresse pós-traumático. Em I. Andretta \& M. S. Oliveira (Eds.), Manual prático de terapia cognitivo-comportamental (pp. 303-318). São Paulo: Casa do Psicólogo.

Laposa, J. M., \& Alden, L. E. (2003). Posttraumatic stress disorder in the emergency room: Exploration of a cognitive model. Behaviour Research and Therapy, 41, 49-65.

McNally, R. J. (2003). Remembering trauma. Cambridge, MA: Belknap/Harvard.

Moser, J. S., Cahill, S. P., \& Foa, E. B. (2010). Evidence for poorer outcome in patients with severe negative trauma-related cognitions receiving prolonged exposure plus cognitive restructuring: Implications for treatment matching in posttraumatic stress disorder. The Journal of Nervous and Mental Disease, 198, 72-75.
Mowrer, O. H. (1960). Learning theory and the symbolic processes. New York: Wiley.

O’Donnell, M. L., Creamer, M., Elliott, P., Bryant, R., McFarlane, A., \& Silove, D. (2009). Prior trauma and psychiatric history as risk factors for intentional and unintentional injury in Australia. The Journal of Trauma, Injury, Infection, and Critical Care, $66,470-476$.

Ozer, E. J., Best, S. R., Lipsey, T. L., \& Weiss, D. S. (2003). Predictors of posttraumatic stress disorder and symptoms in adults: A meta-analysis. Psychological Bulletin, 129, 52-73.

Ozer, E. J., \& Weiss, D. S. (2004). Who develops posttraumatic stress disorder? American Psychological Society, 13, 169-172.

Riggs, D. S., Cahill, S. P., \& Foa, E. B. (2006). Prolonged exposure treatment of posttraumatic stress disorder. Em V. M. Follette \& J. I. Ruzek (Eds.), Cognitive-behavioral therapies for trauma (pp. 65-95). New York: Guilford Press.

Rothbaum, B. O., Meadows, E. A., Resick, P., \& Foy, D. W. (2000). Cognitive-behavioral therapy. Em E. B. Foa, T. M. Keane \& M. J. Friedman (Eds.), Effective treatments for PTSD (pp. 60-83). New York: Guilford Press.

Schiraldi, G. R. (1999). The post-traumatic stress disorder sourcebook: A guide to healing, recovery, and growth. New York: McGraw-Hill.

Stallard, P., \& Smith, E. (2007). Appraisals and cognitive coping styles associated with chronic post-traumatic symptoms in child road traffic accident survivors. Journal of Child Psychology and Psychiatry, 48, 194-201.

Taylor, S. (2006). Clinican's guide to PTSD: A cognitivebehavioral approach. New York: Guilford Press.

Yehuda, R. (1999). Risk factors for posttraumatic stress disorder. Washington, DC: American Psychiatric Press.
Recebido: 13/10/2010 Última revisão: 01/05/2012 Aceito: $28 / 06 / 2012$ 